\title{
Effect of Constrained Donor Atom Orientation upon the Stabilities, Complexation Kinetics, Redox Potentials and Structures of Macrocyclic Polythiaether Complexes. Copper(II) Complexes with Cyclopentanediyl Derivatives of [14]aneS 4 in $80 \%$ Methanol
}

Safaa H. Kakos, Luke T. Dressel, Jeffery D. Bushendorf, Casimir P. Kotarba, Prabodha Wijetunge, Chandrika P. Kulatilleke, Michael P. McGillivary, Gezahegn Chaka, Mary Jane Heeg, L. A. Ochrymowycz, and D. B. Rorabacher

Departments of Chemistry, Wayne State University, Detroit, MI 48202, and the University of Wisconsin-Eau Claire, Eau Claire, WI 54701

\section{SUPPORTING INFORMATION}

\section{CONTENTS}

Table S-1 Kinetic Data for the Formation of $\mathrm{Cu}^{\mathrm{II}}($ cis-cypt-[14]aneS 4$)$ in $80 \%$ Methanol at 25 ${ }^{\circ} \mathrm{C}, \mu=0.10\left(\mathrm{ClO}_{4}^{-}\right)$

Table S-2 Kinetic Data for the Formation of $\mathrm{Cu}^{\mathrm{II}}($ trans-cypt-[14]aneS 4$)$ in $80 \%$ Methanol at $25^{\circ} \mathrm{C}, \mu=0.10\left(\mathrm{ClO}_{4}^{-}\right)$

Table S-3 Kinetic Data for the Formation of $\mathrm{Cu}^{\mathrm{II}}\left(\right.$ syn-cis,cis-dicypt-[14]ane $\left.\mathrm{S}_{4}\right)$ in $80 \%$ Methanol at $25{ }^{\circ} \mathrm{C}, \mu=0.10\left(\mathrm{ClO}_{4}^{-}\right)$

Table S-4 Kinetic Data for the Formation of $\mathrm{Cu}^{\mathrm{II}}($ anti-cis,cis-dicypt-[14]aneS 4$)$ in 80\% Methanol at $25{ }^{\circ} \mathrm{C}, \mu=0.10\left(\mathrm{ClO}_{4}^{-}\right)$

Table S-5 Kinetic Data for the Formation of $\mathrm{Cu}^{\mathrm{II}}($ meso-trans,trans-dicypt-[14]aneS 4$)$ in $80 \%$ Methanol at $25{ }^{\circ} \mathrm{C}, \mu=0.10\left(\mathrm{ClO}_{4}^{-}\right)$

Table S-6 Kinetic Data for the Formation of $\mathrm{Cu}^{\mathrm{II}}\left(\right.$ dl-trans,trans-dicypt-[14]ane $\left.\mathrm{S}_{4}\right)$ in $80 \%$ Methanol at $25{ }^{\circ} \mathrm{C}, \mu=0.10\left(\mathrm{ClO}_{4}^{-}\right)$

Table S-7 Kinetic Data for the Formation of $\mathrm{Cu}^{\mathrm{II}}($ cis,trans-dicypt-[14]aneS 4$)$ in $80 \%$ Methanol at $25{ }^{\circ} \mathrm{C}, \mu=0.10\left(\mathrm{ClO}_{4}^{-}\right)$

Table S-8 Kinetic Data for the Displacement of $\mathrm{Cu}^{\mathrm{II}}$ (cis-cypt-[14]aneS 4 ) by Mercury(II) Ion in $80 \%$ Methanol at $25^{\circ} \mathrm{C}, \mu=0.10\left(\mathrm{ClO}_{4}^{-}\right)$ 
Table S-9 Kinetic Data for the Displacement of $\mathrm{Cu}^{\mathrm{II}}\left(\right.$ trans-cypt-[14]aneS $\left.\mathrm{S}_{4}\right)$ by Mercury(II) Ion in $80 \%$ Methanol at $25{ }^{\circ} \mathrm{C}, \mu=0.10\left(\mathrm{ClO}_{4}^{-}\right)$

Table S-10 Kinetic Data for the Displacement of $\mathrm{Cu}^{\mathrm{II}}\left(\right.$ syn-cis,cis-dicypt-[14] $\left.\mathrm{aneS}_{4}\right)$ by Mercury(II) Ion in $80 \%$ Methanol at $25^{\circ} \mathrm{C}, \mu=0.10\left(\mathrm{ClO}_{4}^{-}\right)$

Table S-11 Kinetic Data for the Displacement of $\mathrm{Cu}^{\mathrm{II}}$ (anti-cis,cis-dicypt-[14]aneS 4 ) by Mercury(II) Ion in $80 \%$ Methanol at $25^{\circ} \mathrm{C}, \mu=0.10\left(\mathrm{ClO}_{4}^{-}\right)$

Table S-12 Kinetic Data for the Displacement of $\mathrm{Cu}^{\mathrm{II}}$ (meso-trans,trans-dicypt-[14]aneS 4 ) by Mercury(II) Ion in $80 \%$ Methanol at $25^{\circ} \mathrm{C}, \mu=0.10\left(\mathrm{ClO}_{4}^{-}\right)$

Table S-13 Kinetic Data for the Displacement of $\mathrm{Cu}^{\mathrm{II}}($ dl-trans,trans-dicypt-[14]aneS 4 ) by Mercury(II) Ion in $80 \%$ Methanol at $25^{\circ} \mathrm{C}, \mu=0.10\left(\mathrm{ClO}_{4}^{-}\right)$

Table S-14 Kinetic Data for the Displacement of $\mathrm{Cu}^{\mathrm{II}}\left(\right.$ cis, trans-dicypt-[14]aneS $\left.\mathrm{S}_{4}\right)$ by Mercury(II) Ion in $80 \%$ Methanol at $25^{\circ} \mathrm{C}, \mu=0.10\left(\mathrm{ClO}_{4}^{-}\right)$

Table S-15 Comparison of $k_{\mathrm{Hg}}$ Values for Copper(II) Complexes with Cyclopentanediyl and Cyclohexanediyl Derivatives of [14] aneS 4 in $80 \%$ Methanol at $25^{\circ} \mathrm{C}, \mu=0.10$ $\left(\mathrm{ClO}_{4}^{-}\right)$ 
Table S-1. Kinetic Data for the Formation of $\mathrm{Cu}^{\mathrm{II}}($ cis-cypt-[14]aneS 4$)$ in $80 \%$ Methanol at $25{ }^{\circ} \mathrm{C}$, $\mu=0.10\left(\mathrm{ClO}_{4}^{-}\right) . \mathrm{C}_{\mathrm{L}}=32.1 \mu \mathrm{M}$.

\begin{tabular}{cccc}
\hline $\mathrm{C}_{\mathrm{Cu}(\mathrm{II}), \mathrm{mM}}$ & $k_{\mathrm{obs}}, \mathrm{s}^{-1}$ & $\mathrm{C}_{\mathrm{Cu}(\mathrm{II})}, \mu \mathrm{M}$ & $k_{\mathrm{obs}}, \mathrm{s}^{-1}$ \\
\hline 0.974 & $23.0(3)$ & 5.84 & $128(17)$ \\
1.95 & $40.9(17)$ & 7.79 & $171(22)$ \\
3.90 & $81.7(28)$ & &
\end{tabular}

$a$ Values in parentheses in this and the following tables represent the standard deviation in terms of the last digits listed. Thus, 23.0(3) and 171(22) represent 23.0 \pm 0.3 ; and $171 \pm 22$, respectively.

Table S-2. Kinetic Data for the Formation of $\mathrm{Cu}^{\mathrm{II}}($ trans-cypt-[14]aneS 4$)$ in $80 \%$ Methanol at 25 ${ }^{\circ} \mathrm{C}, \mu=0.10\left(\mathrm{ClO}_{4}^{-}\right) . \mathrm{C}_{\mathrm{L}}=34.2 \mu \mathrm{M}$.

\begin{tabular}{cccc}
\hline $\mathrm{C}_{\mathrm{Cu}(\mathrm{II}), \mathrm{mM}}$ & $k_{\mathrm{obs}}, \mathrm{s}^{-1}$ & $\mathrm{C}_{\mathrm{Cu}(\mathrm{II})}, \mu \mathrm{M}$ & $k_{\mathrm{obs}}, \mathrm{s}^{-1}$ \\
\hline 0.974 & $30.8(28)$ & 5.84 & $53.0(30)$ \\
1.95 & $34.6(15)$ & 7.79 & $69.7(33)$ \\
3.90 & $44.4(27)$ & &
\end{tabular}

Table S-3. Kinetic Data for the Formation of $\mathrm{Cu}^{\mathrm{II}}($ syn-cis,cis-dicypt-[14]aneS 4$)$ in $80 \%$ Methanol at $25{ }^{\circ} \mathrm{C}, \mu=0.10\left(\mathrm{ClO}_{4}^{-}\right) . \mathrm{C}_{\mathrm{L}}=31.5 \mu \mathrm{M}$.

\begin{tabular}{cccc}
\hline $\mathrm{C}_{\mathrm{Cu}(\mathrm{II}), \mathrm{mM}}$ & $k_{\mathrm{obs}}, \mathrm{s}^{-1}$ & $\mathrm{C}_{\mathrm{Cu}(\mathrm{II}), \mu \mathrm{M}}$ & $k_{\mathrm{obs}}, \mathrm{s}^{-1}$ \\
\hline 0.967 & $48.8(8)$ & 5.80 & $279(23)$ \\
1.94 & $93.4(22)$ & 7.74 & $396(46)$ \\
3.87 & $191(16)$ & &
\end{tabular}


Table S-4. Kinetic Data for the Formation of $\mathrm{Cu}^{\mathrm{II}}($ syn-cis,cis-dicypt-[14]aneS 4$)$ in $80 \%$ Methanol at $25^{\circ} \mathrm{C}, \mu=0.10\left(\mathrm{ClO}_{4}^{-}\right) . \mathrm{C}_{\mathrm{L}}=19.7 \mu \mathrm{M}$.

\begin{tabular}{cccc}
\hline $\mathrm{C}_{\mathrm{Cu}(\mathrm{II}), \mathrm{mM}}$ & $k_{\mathrm{obs}}, \mathrm{s}^{-1}$ & $\mathrm{C}_{\mathrm{Cu}(\mathrm{II})}, \mu \mathrm{M}$ & $k_{\mathrm{obs},}, \mathrm{s}^{-1}$ \\
\hline 0.974 & $30.1(15)$ & 5.84 & $96.2(160)$ \\
1.95 & $42.9(23)$ & 7.79 & $128(9)$ \\
3.90 & $70.3(57)$ & &
\end{tabular}

Table S-5. Kinetic Data for the Formation of $\mathrm{Cu}^{\mathrm{II}}$ (meso-trans,trans-dicypt-[14]aneS 4 ) in 80\% Methanol at $25^{\circ} \mathrm{C}, \mu=0.10\left(\mathrm{ClO}_{4}^{-}\right) . \mathrm{C}_{\mathrm{L}}=30.8 \mu \mathrm{M}$.

\begin{tabular}{cccc}
\hline $\mathrm{C}_{\mathrm{Cu}(\mathrm{II}), \mathrm{mM}}$ & $k_{\mathrm{obs}}, \mathrm{s}^{-1}$ & $\mathrm{C}_{\mathrm{Cu}(\mathrm{II})}, \mu \mathrm{M}$ & $k_{\mathrm{obs}}, \mathrm{s}^{-1}$ \\
\hline 0.974 & $2.13(4)$ & 5.84 & $3.97(9)$ \\
1.95 & $2.46(4)$ & 7.79 & $4.53(6)$ \\
3.90 & $3.27(4)$ & &
\end{tabular}

Table S-6. Kinetic Data for the Formation of $\mathrm{Cu}^{\mathrm{II}}\left(\right.$ dl-trans,trans-dicypt-[14]aneS $\left.\mathrm{S}_{4}\right)$ in $80 \%$ Methanol at $25^{\circ} \mathrm{C}, \mu=0.10\left(\mathrm{ClO}_{4}^{-}\right) . \mathrm{C}_{\mathrm{L}}=31.5 \mu \mathrm{M}$.

\begin{tabular}{cccc}
\hline $\mathrm{C}_{\mathrm{Cu}(\mathrm{II}), \mathrm{mM}}$ & $k_{\mathrm{obs}}, \mathrm{s}^{-1}$ & $\mathrm{C}_{\mathrm{Cu}(\mathrm{II}), \mu \mathrm{M}}$ & $k_{\mathrm{obs}}, \mathrm{s}^{-1}$ \\
\hline 24.2 & $113(8)$ & 96.7 & $198(22)$ \\
48.4 & $124(6)$ & 145 & $262(31)$ \\
72.6 & $167(13)$ & &
\end{tabular}


Table S-7. Kinetic Data for the Formation of $\mathrm{Cu}^{\mathrm{II}}($ cis,trans-dicypt-[14]aneS 4$)$ in $80 \%$ Methanol at $25^{\circ} \mathrm{C}, \mu=0.10\left(\mathrm{ClO}_{4}^{-}\right) . \mathrm{C}_{\mathrm{L}}=31.5 \mu \mathrm{M} . \quad \mathrm{C}_{\mathrm{L}}=29.7 \mu \mathrm{M}$.

\begin{tabular}{cccc}
\hline $\mathrm{C}_{\mathrm{Cu}(\mathrm{II}), \mathrm{mM}}$ & $k_{\mathrm{obs}}, \mathrm{s}^{-1}$ & $\mathrm{C}_{\mathrm{Cu}(\mathrm{II})}, \mu \mathrm{M}$ & $k_{\mathrm{obs}}, \mathrm{s}^{-1}$ \\
\hline 0.974 & $18.2(4)$ & 5.84 & $58.0(12)$ \\
1.95 & $24.3(5)$ & 7.79 & $76.3(22)$ \\
3.90 & $41.6(8)$ & &
\end{tabular}

Table S-8. Kinetic Data for the Displacement of $\mathrm{Cu}^{\mathrm{II}}($ cis-cypt-[14]aneS 4$)$ by Mercury(II) Ion in $80 \%$ Methanol at $25{ }^{\circ} \mathrm{C}, \mu=0.10\left(\mathrm{ClO}_{4}^{-}\right) . \mathrm{C}_{\mathrm{L}}=32.1 \mu \mathrm{M}$.

\begin{tabular}{cccc}
\hline $\mathrm{C}_{\mathrm{Hg}(\mathrm{II}), \mathrm{mM}}$ & $k_{\mathrm{obs}}, \mathrm{s}^{-1}$ & $\mathrm{C}_{\mathrm{Hg}(\mathrm{II})}, \mu \mathrm{M}$ & $k_{\mathrm{obs}}, \mathrm{s}^{-1}$ \\
\hline 0.297 & $2.06(4)$ & 0.594 & $2.49(8)$ \\
0.396 & $2.20(5)$ & 0.792 & $3.09(12)$ \\
0.495 & $2.44(7)$ & &
\end{tabular}

Table S-9. Kinetic Data for the Displacement of $\mathrm{Cu}^{\mathrm{II}}$ (trans-cypt-[14]aneS 4 ) by Mercury(II) Ion in $80 \%$ Methanol at $25^{\circ} \mathrm{C}, \mu=0.10\left(\mathrm{ClO}_{4}^{-}\right) . \mathrm{C}_{\mathrm{L}}=32.1 \mu \mathrm{M}$.

\begin{tabular}{cccc}
\hline $\mathrm{C}_{\mathrm{Hg}(\mathrm{II}), \mathrm{mM}}$ & $k_{\mathrm{obs}}, \mathrm{s}^{-1}$ & $\mathrm{C}_{\mathrm{Hg}(\mathrm{II})}, \mu \mathrm{M}$ & $k_{\mathrm{obs}}, \mathrm{s}^{-1}$ \\
\hline 0.297 & $26.2(9)$ & 0.594 & $31.2(3)$ \\
0.396 & $27.9(10)$ & 0.792 & $33.4(7)$ \\
0.495 & $30.1(9)$ & &
\end{tabular}


Table S-10. Kinetic Data for the Displacement of $\mathrm{Cu}^{\mathrm{II}}\left(\right.$ syn-cic,cis-dicypt-[14] $\left.\mathrm{aneS}_{4}\right)$ by Mercury(II) Ion in $80 \%$ Methanol at $25{ }^{\circ} \mathrm{C}, \mu=0.10\left(\mathrm{ClO}_{4}^{-}\right) . \mathrm{C}_{\mathrm{L}}=31.5 \mu \mathrm{M}$.

\begin{tabular}{cccc}
\hline $\mathrm{C}_{\mathrm{Hg}(\mathrm{II}), \mathrm{mM}}$ & $k_{\mathrm{obs}}, \mathrm{s}^{-1}$ & $\mathrm{C}_{\mathrm{Hg}(\mathrm{II})}, \mu \mathrm{M}$ & $k_{\mathrm{obs}}, \mathrm{s}^{-1}$ \\
\hline 0.297 & $0.163(7)$ & 0.594 & $0.291(20)$ \\
0.396 & $0.175(3)$ & 0.792 & $0.319(19)$ \\
0.495 & $0.194(7)$ & &
\end{tabular}

Table S-11. Kinetic Data for the Displacement of $\mathrm{Cu}^{\mathrm{II}}$ (anti-cic,cis-dicypt-[14] $\mathrm{aneS}_{4}$ ) by Mercury(II) Ion in $80 \%$ Methanol at $25{ }^{\circ} \mathrm{C}, \mu=0.10\left(\mathrm{ClO}_{4}^{-}\right) . \mathrm{C}_{\mathrm{L}}=19.7 \mu \mathrm{M}$.

\begin{tabular}{cccc}
\hline $\mathrm{C}_{\mathrm{Hg}(\mathrm{II}), \mathrm{mM}}$ & $k_{\mathrm{obs},}, \mathrm{s}^{-1}$ & $\mathrm{C}_{\mathrm{Hg}(\mathrm{II})}, \mu \mathrm{M}$ & $k_{\mathrm{obs}}, \mathrm{s}^{-1}$ \\
\hline 0.247 & $10.6(2)$ & 1.24 & $11.1(2)$ \\
0.495 & $9.93(12)$ & 1.98 & $10.6(2)$ \\
0.742 & $10.5(2)$ & &
\end{tabular}

Table S-12. Kinetic Data for the Displacement of $\mathrm{Cu}^{\mathrm{II}}$ (meso-trans, trans-dicypt-[14]aneS 4 ) by Mercury(II) Ion in $80 \%$ Methanol at $25{ }^{\circ} \mathrm{C}, \mu=0.10\left(\mathrm{ClO}_{4}^{-}\right) . \mathrm{C}_{\mathrm{L}}=30.8 \mu \mathrm{M}$.

\begin{tabular}{cccc}
\hline $\mathrm{C}_{\mathrm{Hg}(\mathrm{II}), \mathrm{mM}}$ & $k_{\mathrm{obs}}, \mathrm{s}^{-1}$ & $\mathrm{C}_{\mathrm{Hg}(\mathrm{II})}, \mu \mathrm{M}$ & $k_{\mathrm{obs}}, \mathrm{s}^{-1}$ \\
\hline 0.297 & $1.87(2)$ & 0.594 & $2.14(4)$ \\
0.396 & $2.00(3)$ & 0.792 & $2.46(5)$ \\
0.495 & $2.08(5)$ & &
\end{tabular}


Table S-13. Kinetic Data for the Displacement of $\mathrm{Cu}^{\mathrm{II}}($ dl-trans,trans-dicypt-[14]aneS 4$)$ by Mercury(II) Ion in $80 \%$ Methanol at $25^{\circ} \mathrm{C}, \mu=0.10\left(\mathrm{ClO}_{4}^{-}\right) . \mathrm{C}_{\mathrm{L}}=31.5 \mu \mathrm{M}$.

\begin{tabular}{cccc}
\hline $\mathrm{C}_{\mathrm{Hg}(\mathrm{II}), \mathrm{mM}}$ & $k_{\mathrm{obs}}, \mathrm{s}^{-1}$ & $\mathrm{C}_{\mathrm{Hg}(\mathrm{II})}, \mu \mathrm{M}$ & $k_{\mathrm{obs}}, \mathrm{s}^{-1}$ \\
\hline 0.297 & $75.1(28)$ & 0.594 & $76.5(27)$ \\
0.396 & $77.0(13)$ & 0.792 & $81.7(25)$ \\
0.495 & $75.7(20)$ & &
\end{tabular}

Table S-14. Kinetic Data for the Displacement of $\mathrm{Cu}^{\mathrm{II}}($ cis,trans-dicypt-[14]aneS 4$)$ by Mercury(II) Ion in $80 \%$ Methanol at $25^{\circ} \mathrm{C}, \mu=0.10\left(\mathrm{ClO}_{4}^{-}\right) . \mathrm{C}_{\mathrm{L}}=29.7 \mu \mathrm{M}$.

\begin{tabular}{cccc}
\hline $\mathrm{C}_{\mathrm{Hg}(\mathrm{II}), \mathrm{mM}}$ & $k_{\mathrm{obs}}, \mathrm{s}^{-1}$ & $\mathrm{C}_{\mathrm{Hg}(\mathrm{II})}, \mu \mathrm{M}$ & $k_{\mathrm{obs}}, \mathrm{s}^{-1}$ \\
\hline 0.297 & $9.83(10)$ & 0.594 & $12.2(2)$ \\
0.396 & $10.5(1)$ & 0.792 & $15.9(4)$ \\
0.495 & $11.5(2)$ & &
\end{tabular}

Table S-15. Comparison of $k_{\mathrm{Hg}}$ Values for Copper(II) Complexes with Cyclopentanediyl and Cyclohexanediyl Derivatives of [14]aneS 4 in $80 \%$ Methanol at $25^{\circ} \mathrm{C}, \mu=0.10\left(\mathrm{ClO}_{4}^{-}\right)$

\begin{tabular}{llclcc}
\hline $\begin{array}{l}\text { Cycloalkyl } \\
\text { Substituents }\end{array}$ & \multicolumn{1}{c}{$\begin{array}{c}k_{\mathrm{d}}, \mathrm{s}^{-1} \\
\text { cyhx series } a\end{array}$} & $\begin{array}{c}k_{\mathrm{Hg}}, \mathrm{M}^{-1} \mathrm{~s}^{-1} \\
\text { cypt series }\end{array}$ & cyhx series $a$ & \multicolumn{2}{c}{$10^{3} k_{\mathrm{d}} / k_{\mathrm{Hg}}, \mathrm{M}$} \\
\hline cypt series & cyhx series \\
parent ligand (L0) & 8.6 & \multicolumn{2}{c}{821} & 0.83 & 34 \\
cis & 0.18 & $1.8(2) \times 10^{3}$ & $52(2)$ & 0.62 & 12
\end{tabular}




$\begin{array}{llllll}\text { syn-cis,cis } & 0.0023 & 0.29(8) \times 10^{3} & 0.27(4) & 0.21 & 8.5 \\ \text { anti-cis,cis } & 0.50 & b & 19(2) & b & 26 \\ \text { meso-trans,trans } & 0.0066 & 1.07(9) \times 10^{3} & 3.0(1) & 1.4 & 22 \\ \text { dl-trans,trans } & \approx 0.01-0.02 & 12(4) \times 10^{3} & b & 5.9 & b \\ \text { cis,trans } & 0.011 & 10(1) \times 10^{3} & 2.8(2) & 0.67 & 4.0\end{array}$

${ }^{a}$ Ref Q12. $b$ The slope based on eq 8 was not statistically significant. 